\title{
SCATTERING OF MEMBRANE COUPLED GRAVITY WAVES BY PARTIAL VERTICAL BARRIERS
}

\author{
S. R. MANAM ${ }^{1}$ \\ (Received 26 July, 2009; revised 20 January, 2010)
}

\begin{abstract}
Scattering of membrane coupled gravity waves in deep water by partial vertical barriers is investigated by the recently developed expansion formulae for wave structure interaction problems. The horizontal thin membrane is considered to be under uniform tension and is covering the free surface. The analysis is based on the linearized theory of water waves, and by combining the kinematic and dynamic conditions at the membrane covered surface, one may derive a not so well-posed mixed boundary value problem for Laplace's equation with third-order boundary condition. The flexible membrane is attached by a spring to the surface piercing barrier, giving suitable edge conditions for the unique solution. The boundary value problem has been converted into dual integral equations with kernels composed of trigonometric functions, which are then solved analytically. The important physical quantities such as reflection and transmission coefficients for both cases of submerged and surface piercing barriers are obtained analytically in terms of modified Bessel functions. It is found that complete reflection or transmission is possible at certain resonant frequencies for the incident membrane coupled waves. Numerical results are plotted and discussed for different values of the nondimensional membrane tension parameter.
\end{abstract}

2000 Mathematics subject classification: primary 74J05; secondary 45F10.

Keywords and phrases: mixed boundary value problem, logarithmic kernels, reflection coefficient, mode-coupling relation.

\section{Introduction}

Design of floating flexible breakwaters to protect offshore structures in deep water has in recent times attracted a lot of attention. Among various wave barriers, flexible membranes have a number of advantages: they are easy to carry, reusable, cost effective, removable and, most importantly, have less environmental impact on coastal processes. They are mainly made up of synthetic fibre, rubber or a polymeric material. Oil booms and slit curtains are examples of membrane structures (Sawaragi [12]). Another advantage of using these floating structures is that they can be used in

\footnotetext{
${ }^{1}$ Department of Mathematics, Indian Institute of Technology Madras, Chennai 600036, India; e-mail: manam@iitm.ac.in.

(C) Australian Mathematical Society 2010, Serial-fee code 1446-1811/2010 \$16.00
} 
unfavourable seabed soil conditions as well. Mathematically, the membrane can be modelled as a tensioned-beam plate for which the bending stiffness approaches zero. In such a situation, the membrane is modelled as a thin and inextensible sheet acting under tension at the interaction boundary where the membrane meets the open water region. Thus, under the assumption that the membrane is of constant and uniform density, its response is governed by the two-dimensional string equation.

Cho and Kim [2] investigated the interaction of oblique waves in finite depth with a submerged horizontal membrane by using a mode expansion method as well as a boundary integral equation method. Other investigations (Sahoo et al. [11], Yip et al. [16], Cho and Kim [3]) in finite depth prove that these floating structures are very effective in reducing wave transmission over a wide range of wave periods. In this context, the study of the scattering of membrane coupled waves by partial vertical barriers placed in the fluid domain may be of interest.

Various methods of solution are explained for different vertical as well as other barrier topographies in free surface wave scattering by Ursell [14], Williams [15] and others. Evans [6] first investigated the surface tension effect on the two-dimensional transmission problem of time-harmonic water waves for partially immersed vertical barriers. Later, Rhodes-Robinson [10] studied the same problem more generally by involving vertical wave-makers. The physically important quantities, such as reflection and transmission of an incident wave, were derived in terms of the edge slope constants which are presumed to be known. However, numerical computation of these quantities was not done since values of these presumed constants have not been found experimentally. When the free surface is covered with a flexible membrane, the governing mixed boundary value problem is the same as that corresponding to the scattering problem involving surface tension for a submerged barrier. Except for the edge conditions specified at the surface edge of the barrier, the equivalence is true even for the case of a surface piercing barrier. Since the velocity across the barrier is not continuous at the intersection point on the surface, the induced unknown constants can be determined from the naturally specified edge constants.

In the present paper, a simple and straightforward method is demonstrated to solve the mixed boundary value problem associated with the scattering of linear membrane coupled gravity waves by partial barriers. These are a completely submerged vertical barrier extended to the bottom of the water column of infinite depth and a finitelength surface piercing barrier. The problem has been reduced to solving a dual integral equation with a kernel comprised of trigonometric functions. It may be remarked that the crucial factor in the reduction process has been the utilization of expansion formulae developed by Manam et al. [9]. The behaviour of one of these dual integral equations, at the point where the boundary condition changes, plays a crucial role in determining their solution. The reduced dual integral equations are solved subsequently with the aid of a weakly singular integral equation (see Chakrabarti and Manam [1] and Estrada and Kanwal, [5]). The method forces its solution to be bounded at both end points and that brings out certain conditions to be satisfied by the forcing function of the integral equation. 
The mathematical description of the mixed boundary value problem is given in Section 2. The reduction of the boundary value problem for the two barrier configurations into dual integral equations and their analytical solution procedure are described in Sections 3.1 and 3.2. The numerical results for the reflection coefficient of an incident membrane coupled gravity wave are discussed in Section 3.3.

\section{Mathematical formulation}

A general mixed boundary value problem modelling membrane coupled gravity wave scattering by partial vertical barriers is formulated under the assumption of linearized water wave theory and small amplitude membrane response. The fluid domain is assumed to be two-dimensional in nature and the floating membrane is considered as a one-dimensional string.

The two-dimensional Cartesian coordinate system is used in which the $y$-axis is taken vertically downward so that $y>0, x \in \mathbb{R}$ is the region occupied by the fluid. Assuming that motion is irrotational in an incompressible inviscid fluid under the action of gravity, there exists a velocity potential $\Phi(x, y, t)$ satisfying

$$
\frac{\partial^{2} \Phi}{\partial x^{2}}+\frac{\partial^{2} \Phi}{\partial y^{2}}=0, \quad x \in \mathbb{R}, \quad y>0 .
$$

The linearized kinematic and dynamic boundary conditions on the mean free surface $y=0$ are

$$
\frac{\partial \Phi}{\partial y}=\frac{\partial \eta}{\partial t} \quad \text { and } \quad-P=\rho \frac{\partial \Phi}{\partial t}-\rho g \eta .
$$

Here $\eta$ is the vertical displacement of the membrane, $\rho$ is the constant fluid density, $P$ is the hydrodynamic pressure and $g$ is the acceleration due to gravity. Considering that the membrane is a thin, homogeneous and inextensible sheet with uniform mass $m_{s}\left(m_{s}=\rho_{s} d ; d\right.$ is the thickness of the membrane, $\rho_{s}$ is the uniform membrane mass density) under constant tension $S$, the membrane displacement is related to the differential pressure $P_{S}$ acting on the membrane by

$$
-P_{s}(x, y, t)=-S \frac{\partial^{2} \eta}{\partial x^{2}}+m_{s} \frac{\partial^{2} \eta}{\partial t^{2}} .
$$

Balancing the hydrodynamic pressure $P$ with the sum of the atmospheric pressure (without loss of generality it can be assumed to be zero) and the differential pressure $P_{s}$ on the membrane covered region, and eliminating $\eta$ from the relations (2.2) and (2.3), the boundary condition on the surface $y=0$ is obtained as

$$
S \frac{\partial^{3} \Phi}{\partial y^{3}}+m_{s} \frac{\partial^{3} \Phi}{\partial t^{2} \partial y}+\rho g \frac{\partial \Phi}{\partial y}-\rho \frac{\partial^{2} \Phi}{\partial t^{2}}=0 .
$$

Considering simple-harmonic fluid and membrane motions of an angular frequency $\omega$, the velocity potential and the displacement are represented as $\Phi(x, y, t)=\operatorname{Re}\left\{\phi(x, y) e^{-i \omega t}\right\}$ and $\eta(x, t)=\operatorname{Re}\left\{\zeta(x) e^{-i \omega t}\right\}$. Then the surface 
boundary condition becomes

$$
M \frac{\partial^{3} \phi}{\partial y^{3}}+\frac{\partial \phi}{\partial y}+K \phi=0 \quad \text { on } y=0, x \in \mathbb{R},
$$

where $M=S /\left(\rho g-m_{s} \omega^{2}\right)$ and $K=\rho \omega^{2} /\left(\rho g-m_{s} \omega^{2}\right)$.

Further, we assume that the membrane is elastically supported by a spring of zero vertical stiffness at the mean surface level to the surface piercing barrier (see [8]). Thus, on both sides of the barrier at the surface,

$$
\frac{\partial^{2} \phi}{\partial x \partial y}=0 \quad \text { at }(x, y)=\left(0^{ \pm}, 0\right) .
$$

On the rigid vertical structure occupied at $x=0, y \in(b, \infty)$ or $(0, b)$ with $b>0$, $\phi(x, y)$ satisfies the Neumann boundary condition

$$
\frac{\partial \phi}{\partial x}=0
$$

also known as the condition of vanishing normal velocity.

Also, since the fluid flow is continuous across the gap $x=0, y \in(0, b)$ or $(b, \infty)$, the potential function $\phi(x, y)$ satisfies

$$
\phi\left(0^{-}, y\right)=\phi\left(0^{+}, y\right)
$$

in standard notation and

$$
\phi, \frac{\partial \phi}{\partial x}, \frac{\partial \phi}{\partial y} \rightarrow 0 \quad \text { as } y \rightarrow \infty,
$$

representing no motion as the depth of the fluid becomes large.

The behaviour of $\phi(x, y)$ at infinity in the horizontal direction is given by

$$
\phi(x, y) \rightarrow \begin{cases}e^{i \lambda x-\lambda y}+R e^{-i \lambda x-\lambda y} & \text { as } x \rightarrow-\infty \\ T e^{i \lambda x-\lambda y} & \text { as } x \rightarrow \infty\end{cases}
$$

where $R$ and $T$ are the unknown reflection and transmission coefficients in wave scattering (see Stoker [13]) and $\lambda$ is the positive real root of a dispersion equation $M x^{3}+x-K=0$. Note that the dispersion relation has, for specified values of $M$ and $K$, one positive real root $\lambda$ and complex conjugate roots $\lambda_{1}$ and $\bar{\lambda}_{1}$ with negative real part.

The barrier edge conditions, as required for the energy to be finite in the neighbourhood associated with the flow (see Stoker [13, Section 2.4]), are given by

$$
\frac{\partial \phi}{\partial x}(0, y) \sim O\left(|y-t|^{-1 / 2}\right) \quad \text { as } y \rightarrow t,
$$

where $t=b^{+}$and $t=b^{-}$are the end points of the thin vertical structures under consideration. Thus, the boundary value problem is now well defined for the two physical problems concerning the scattering of thin vertical partial barriers. It may be 

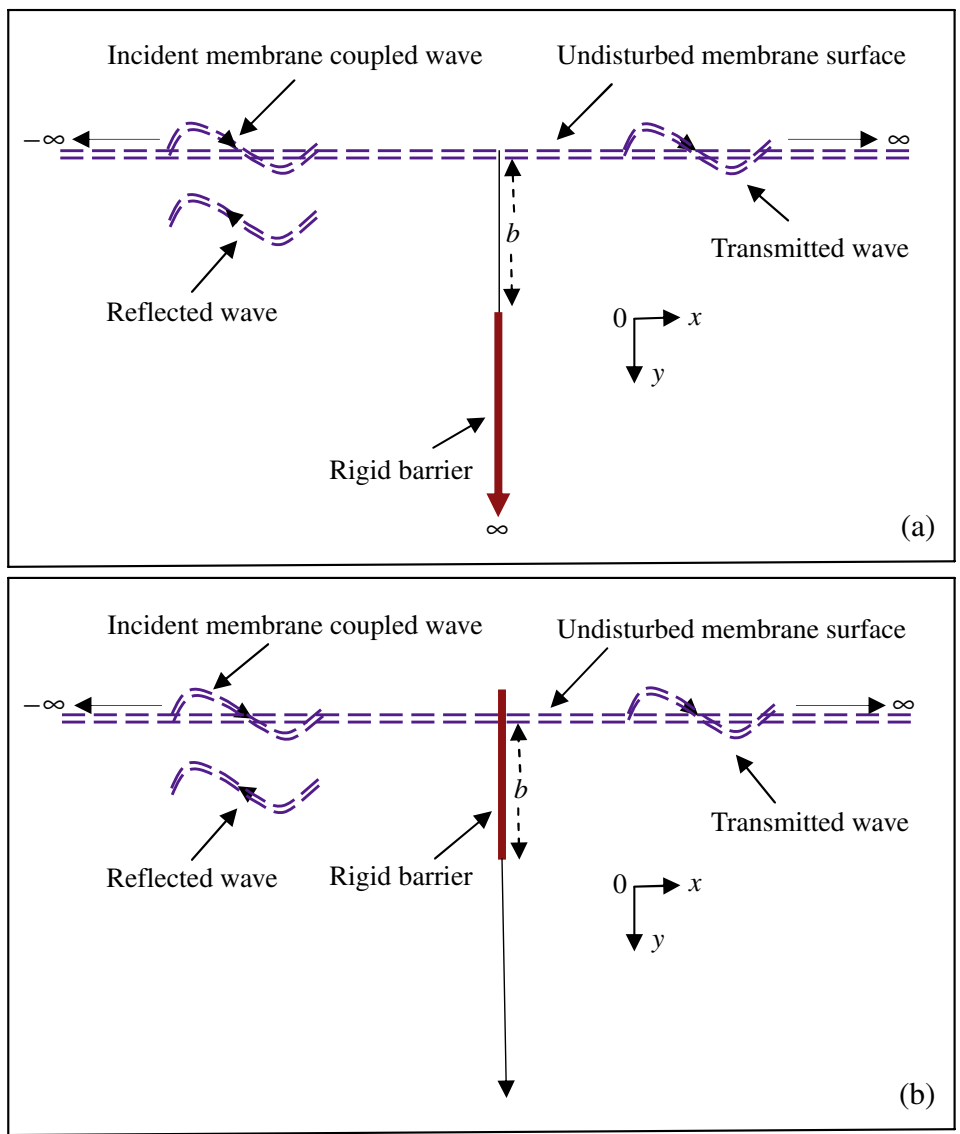

FIGURE 1. Schematic diagram for a flexible membrane covered surface with (a) submerged and (b) surface piercing vertical rigid barrier.

noted that the linearized surface condition for capillary gravity waves is recovered in the relation (2.4) with $m_{s}=0$. It is worth mentioning that the surface tension value is much smaller than the membrane tension value. A schematic diagram is shown in Figure 1 for these physical situations of scattering involving partial vertical barriers.

\section{The method of solution}

It can be shown (Manam et al. [9]) that the unknown velocity potentials $\phi(x, y)$ in the two regions $x>0$ and $x<0$ are expanded as

$$
\phi(x, y)= \begin{cases}T e^{i \lambda x-\lambda y}+\int_{0}^{\infty} B(\xi) L(\xi, y) e^{-\xi x} d \xi, & x>0, \\ e^{i \lambda x-\lambda y}+R e^{-i \lambda x-\lambda y}+\int_{0}^{\infty} A(\xi) L(\xi, y) e^{\xi x} d \xi, & x<0\end{cases}
$$


where $L(\xi, y)=\xi\left(1-M \xi^{2}\right) \cos \xi y-K \sin \xi y, \quad A(\xi)$ and $B(\xi)$ are unknown functions, and $R$ and $T$ are the unknown complex-valued reflected and transmitted amplitudes of the incident wave $e^{i \lambda x-\lambda y}$.

The potential function $\phi(x, y)$ automatically satisfies the partial differential equation (2.1) and conditions (2.4), (2.8) and (2.9) for an appropriate choice of the functions $A(\xi)$ and $B(\xi)$.

In what follows, the solution procedure is given separately for each of the scattering problems involving submerged as well as surface piercing barriers.

3.1. Submerged barrier The problem configuration for this case is sketched in Figure 1(a). Since the horizontal velocity is continuous along the positive $y$-axis, including the point of contact with the flexible membrane, we obtain that

$$
\int_{0}^{\infty} \xi[A(\xi)+B(\xi)] L(\xi, y) d \xi=i \lambda(T+R-1) e^{-\lambda y} \quad \text { for } y \geq 0 .
$$

The functions $L(\xi, y), \xi, y \geq 0$ and $e^{-\lambda y}$ are orthogonal with respect to the modecoupling relation (see Manam et al. [9])

$$
\langle f, g\rangle=\int_{0}^{\infty} f(y) g(y) d y+\frac{M}{K} f^{\prime}(0) g^{\prime}(0),
$$

where / denotes the derivative and hence

$$
T=1-R, \quad A(\xi)=-B(\xi) .
$$

This may also be achieved by equating real and imaginary parts to zero and then by making use of the orthogonal mode-coupling relation. Applying conditions (2.7) and (2.6) to relation (3.1), we derive a pair of integral equations

$$
\begin{array}{cc}
\int_{0}^{\infty} A(\xi) L(\xi, y) d \xi=-R e^{-\lambda y} \quad \text { on } y \in(0, b), \\
\int_{0}^{\infty} \xi A(\xi) L(\xi, y) d \xi=-i \lambda(1-R) e^{-\lambda y} \text { on } y \in(b, \infty),
\end{array}
$$

or alternatively,

$$
\begin{aligned}
& \mathcal{L} \int_{0}^{\infty} A(\xi) \sin \xi y d \xi=-R e^{-\lambda y} \quad \text { on } y \in(0, b), \\
& \mathcal{L} \int_{0}^{\infty} \xi A(\xi) \sin \xi y d \xi=-i \lambda(1-R) e^{-\lambda y} \quad \text { on } y \in(b, \infty),
\end{aligned}
$$

where

$$
\mathcal{L}=\left(M d^{3} / d y^{3}+d / d y-K\right) .
$$

Then (3.2) and (3.3) yield the dual integral equations

$$
\int_{0}^{\infty} A(\xi) \sin \xi y d \xi=C_{1} e^{\lambda y}+C_{2} e^{\lambda_{1} y}+C_{3} e^{\bar{\lambda}_{1} y}+\frac{R e^{-\lambda y}}{Q(\lambda)} \equiv f(y) \quad \text { for } y \in(0, b)
$$


and

$$
\begin{aligned}
\int_{0}^{\infty} \xi A(\xi) \sin \xi y d \xi & =D_{1} e^{\lambda_{1} y}+D_{2} e^{\bar{\lambda}_{1} y}+D_{3} e^{\lambda y}+\frac{i \lambda(1-R)}{Q(\lambda)} e^{-\lambda y} \\
& \equiv h(y) \quad \text { for } y \in(b, \infty) .
\end{aligned}
$$

Here $Q(\lambda)=\lambda\left(1+M \lambda^{2}\right)+K$ and $C_{1}, C_{2}, C_{3}, D_{1}, D_{2}$ and $D_{3}$ are arbitrary constants. As mentioned earlier, $\lambda, \lambda_{1}$ and $\bar{\lambda}_{1}$ are the roots of the dispersion relation $M x^{3}+x-K=0$.

In order to accommodate the point at infinity along the positive $y$-axis, $D_{3}$ in (3.4) is taken to be zero and at the point at zero the function $f(y)$ satisfies $f(0)=f^{\prime \prime}(0)=0$, that is,

$$
C_{1}+C_{2}+C_{3}+\frac{1}{Q(\lambda)} R=0
$$

and

$$
\lambda^{2} C_{1}+\lambda_{1}^{2} C_{2}+\bar{\lambda}_{1}^{2} C_{3}+\frac{\lambda^{2}}{Q(\lambda)} R=0
$$

We now define

$$
\int_{0}^{\infty} \xi A(\xi) \sin \xi y d \xi=g(y) \text { for } y \in(0, b),
$$

where $g(y)$ is an unknown function to be determined. By the Fourier sine transform, relations (3.5) and (3.8) give

$$
A(\xi)=\frac{2}{\pi \xi} \int_{0}^{\infty} P(y) \sin \xi y d y \quad \text { where } P(y)= \begin{cases}g(y), & \text { for } y \in(0, b), \\ h(y), & \text { for } y \in(b, \infty) .\end{cases}
$$

Putting $A(\xi)$ into (3.4) and after utilizing the result (see Gradshteyn and Ryzhik, [7, Equation 3.741(1)])

$$
\int_{0}^{\infty} \frac{\sin \xi y \sin \xi t}{\xi} d \xi=-\frac{1}{2} \log \left|\frac{y-t}{y+t}\right| \text { for } y, t \in(0, \infty)
$$

we observe that $g(y)$ satisfies the weakly singular integral equation (see Chakrabarti and Manam [1])

$$
\frac{1}{\pi} \int_{0}^{b} g(u) \log \left|\frac{u+x}{u-x}\right| d u=f_{1}(x) \quad \text { for } x \in(0, b),
$$

where

$$
f_{1}(x)=f(x)-\frac{1}{\pi} \int_{b}^{\infty} h(t) \log \left|\frac{x+t}{x-t}\right| d t .
$$

The dual integral equations (3.4) and (3.5) can be differentiated twice as they satisfy the third-order differential equation. The result is

$$
\int_{0}^{\infty} \xi^{2} A(\xi) \sin \xi y d \xi=-\frac{d^{2} f}{d y^{2}} \quad \text { for } y \in(0, b)
$$


and

$$
\int_{0}^{\infty} \xi^{3} A(\xi) \sin \xi y d \xi=-\frac{d^{2} h}{d y^{2}} \quad \text { for } y \in(b, \infty) .
$$

Again, since

$$
\int_{0}^{\infty} \xi^{3} A(\xi) \sin \xi y d \xi=-\frac{d^{2} g}{d y^{2}} \quad \text { for } y \in(0, b)
$$

we obtain

$$
\xi^{2} A(\xi)=-\frac{2}{\pi \xi} \int_{0}^{\infty} \frac{d^{2} P}{d y^{2}} \sin \xi y d y .
$$

Substituting the result of (3.12) into relation (3.11), we derive that the function $g_{1}=d^{2} g / d y^{2}$ satisfies

$$
\frac{1}{\pi} \int_{0}^{b} g_{1}(u) \log \left|\frac{u+x}{u-x}\right| d u=f_{2}(x) \quad \text { for } x \in(0, b),
$$

where

$$
f_{2}(x)=\frac{d^{2} f}{d x^{2}}-\frac{1}{\pi} \int_{b}^{\infty} \frac{d^{2} h}{d t^{2}} \log \left|\frac{x+t}{x-t}\right| d t .
$$

In order to solve the integral equation (3.13) completely, we must know the behaviour of the unknown function $g(u)$ at the end point $u=b$.

Letting

$$
\frac{\partial \phi}{\partial x}(0, y)=F(y) \quad \text { for } y \in(0, b)
$$

relation (3.1) gives

$$
\begin{gathered}
\left(M \frac{d^{3}}{d y^{3}}+\frac{d}{d y}-K\right) \int_{0}^{\infty} \xi A(\xi) \sin \xi y d \xi \\
=i \lambda(1-R) e^{-\lambda y}-F(y) \quad y \in(0, b) .
\end{gathered}
$$

Using the behaviour of the function $F(y)$ at the endpoint $u=b$ from relations (2.10) and (3.14), it is found that the unknown functions $g(y)$ and $d^{2} g / d y^{2}$ are bounded at the end point $y=b$. In particular,

$$
\frac{d^{2} g}{d y^{2}} \sim O\left(|y-t|^{1 / 2}\right) \quad \text { as } y \rightarrow b^{-} .
$$

Therefore, the bounded solution of the integral equation (3.10) is given (see [1]) by

$$
g(u)=\frac{2}{\pi} u \sqrt{b^{2}-u^{2}} \int_{0}^{b} \frac{f_{1}^{\prime}(t)}{\sqrt{b^{2}-t^{2}}\left(u^{2}-t^{2}\right)} d t \quad u \in(0, b),
$$

constrained such that

$$
\int_{0}^{b} \frac{f_{1}^{\prime}(t)}{\sqrt{b^{2}-t^{2}}} d t=0
$$


Also, the bounded solution $g_{1}(u)$ of the integral equation (3.13) is

$$
g_{1}(u)=\frac{d^{2} g}{d u^{2}}=\frac{2}{\pi} u \sqrt{b^{2}-u^{2}} \int_{0}^{b} \frac{f_{2}^{\prime}(t)}{\sqrt{b^{2}-t^{2}}\left(u^{2}-t^{2}\right)} d t \quad u \in(0, b),
$$

provided that

$$
\int_{0}^{b} \frac{f_{2}^{\prime}(t)}{\sqrt{b^{2}-t^{2}}} d t=0
$$

Now equating $A(\xi)$ from relations (3.9) and (3.12), we derive, by integration by parts and then by using the bounded property of $g$ at the end points 0 and $b$, conditions to be satisfied by the functions $g$ and $h$ :

$$
h(b)=0 \quad \text { that is, } e^{\lambda_{1} b} D_{1}+e^{\bar{\lambda}_{1} b} D_{2}-\frac{i \lambda}{Q(\lambda)} e^{-\lambda b} R=-\frac{i \lambda}{Q(\lambda)} e^{-\lambda b}
$$

and

$$
g^{\prime}(b)=h^{\prime}(b) .
$$

Conditions (3.15) and (3.17) are now expressed as

$$
\begin{aligned}
& \lambda J_{1}(\lambda) C_{1}+\lambda_{1} J_{1}\left(\lambda_{1}\right) C_{2}+\bar{\lambda}_{1} J_{1}\left(\bar{\lambda}_{1}\right) C_{3}-K_{0}\left(-b \lambda_{1}\right) D_{1}-K_{0}\left(-b \bar{\lambda}_{1}\right) D_{2} \\
& \quad-\left[\frac{\lambda}{Q(\lambda)} J_{1}(-\lambda)+\frac{i \lambda}{Q(\lambda)} K_{0}(b \lambda)\right] R=\frac{i \lambda}{Q(\lambda)} K_{0}(b \lambda)
\end{aligned}
$$

and

$$
\begin{aligned}
& \lambda^{3} J_{1}(\lambda) C_{1}+\lambda_{1}^{3} J_{1}\left(\lambda_{1}\right) C_{2}+\bar{\lambda}_{1}^{3} J_{1}\left(\bar{\lambda}_{1}\right) C_{3}-\lambda_{1}^{2} K_{0}\left(-b \lambda_{1}\right) D_{1}-\bar{\lambda}_{1}^{2} K_{0}\left(-b \bar{\lambda}_{1}\right) D_{2} \\
& \quad-\left[\frac{\lambda^{3}}{Q(\lambda)} J_{1}(-\lambda)+\frac{i \lambda^{3}}{Q(\lambda)} K_{0}(b \lambda)\right] R=\frac{i \lambda^{3}}{Q(\lambda)} K_{0}(b \lambda),
\end{aligned}
$$

where $K_{0}(x)$ is the modified Bessel function and $J_{i}(x), i=1,2,3,4$, are given in the Appendix.

At this stage, the unknown constants $C_{1}, C_{2}, C_{3}, D_{1}, D_{2}$ and $R$ can be determined, in principle, from relations (3.6), (3.7), and (3.18)-(3.21). However, condition (3.19) is not in a suitable form to express as a linear equation in unknowns with computable coefficients.

In order to modify condition (3.19) to a suitable form, we integrate (3.16) and make use of some of the integrals in the Appendix to get

$$
-b g^{\prime}(b)=\int_{0}^{b} \frac{t^{2} f^{\prime \prime \prime}(t)}{\sqrt{b^{2}-t^{2}}} d t-\frac{4}{\pi^{2}} \int_{b}^{\infty} \frac{t^{2} h^{\prime \prime}(t)}{\sqrt{t^{2}-b^{2}}} d t+\frac{4}{\pi^{2}} \int_{b}^{\infty} t h^{\prime \prime}(t) d t .
$$

Hence (3.19) and (3.22) together produce a linear equation in a computable form as given by

$$
\begin{gathered}
\lambda^{3} Q_{1}(\lambda) C_{1}+\lambda_{1}^{3} Q_{1}\left(\lambda_{1}\right) C_{2}+\bar{\lambda}_{1}^{3} Q_{1}\left(\bar{\lambda}_{1}\right) C_{3}-\lambda_{1} Q_{2}\left(\lambda_{1}\right) D_{1}-\bar{\lambda}_{1} Q_{2}\left(\bar{\lambda}_{1}\right) D_{2} \\
-\frac{\lambda^{2}}{Q(\lambda)}\left[\lambda Q_{1}(-\lambda)-i Q_{3}(-\lambda)\right] R=\frac{i \lambda^{2}}{Q(\lambda)}\left[Q_{3}(\lambda)+\lambda K_{0}(b \lambda)\right],
\end{gathered}
$$


where

$$
\begin{aligned}
& Q_{1}(x)=J_{2}(x)-\frac{1}{2} J_{1}(x), \\
& Q_{2}(x)=x\left\{\frac{4}{\pi^{2}} J_{3}(x)+\frac{1}{2} K_{0}(-b x)\right\}-b e^{x b}\left(1-\frac{4}{\pi^{2}}\right), \\
& Q_{3}(x)=x\left\{\frac{4}{\pi^{2}} J_{3}(-x)-\frac{1}{2} K_{0}(b x)\right\}+b e^{-x b}\left(1-\frac{4}{\pi^{2}}\right)
\end{aligned}
$$

and $J_{i}(x), i=1,2,3,4$, are given in the Appendix.

Thus, the case of scattering of membrane coupled waves by a submerged vertical barrier is completely solved.

3.2. Surface piercing barrier We now turn to the fully analytical solution for the boundary value problem corresponding to scattering of waves by a barrier cutting through the membrane covered surface. The physical problem in this case is sketched in Figure 1(b).

It is a remarkable that the horizontal velocity is not continuous at the intersection point of the membrane covered surface and the rigid barrier. So, the difference in the velocity component on both sides of the interface $x=0$ may be obtained as

$$
\begin{aligned}
\int_{0}^{\infty} \xi & {[A(\xi)+B(\xi)] L(\xi, y) d \xi } \\
& =\left(\phi_{x}\left(0^{-}, y\right)-\phi_{x}\left(0^{+}, y\right)\right)+i \lambda(T+R-1) e^{-\lambda y} \quad \text { for } y>0 .
\end{aligned}
$$

Applying the orthogonal mode-coupling relation on the functions $L(\xi, y), \xi, y>0$ and $e^{-\lambda y}$ (see [9]), we find that

$$
T=1-R+\frac{2 i M\left(\mu^{+}-\mu^{-}\right)}{1+3 M \lambda^{2}}, \quad B(\xi)=-A(\xi)-\frac{2 M\left(\mu^{+}-\mu^{-}\right)}{\pi \Delta(\xi)},
$$

where $\Delta(\xi)=\xi^{2}\left(1-M \xi^{2}\right)^{2}+K^{2}$ and the constants $\mu^{ \pm}=\phi_{x y}\left(0^{ \pm}, 0\right)=0$, which are determined from the edge conditions (2.5).

In this case, conditions (2.7) and (2.6) along with relations (3.1) give rise to a pair of integral equations

$$
\begin{aligned}
\mathcal{L} \int_{0}^{\infty} \xi A(\xi) \sin \xi y d \xi & =-i \lambda(1-R) e^{-\lambda y} & & \text { on } y \in(0, b), \\
\mathcal{L} \int_{0}^{\infty} A(\xi) \sin \xi y d \xi & =-R e^{-\lambda y} & & \text { on } y \in(b, \infty) .
\end{aligned}
$$

Solving (3.24) and (3.25) yields

$$
\begin{aligned}
\int_{0}^{\infty} \xi A(\xi) \sin \xi y d \xi & =C_{1} e^{\lambda y}+C_{2} e^{\lambda_{1} y}+C_{3} e^{\bar{\lambda}_{1} y}+\frac{i \lambda(1-R)}{Q(\lambda)} e^{-\lambda y} \\
& \equiv f(y) \quad \text { for } y \in(0, b)
\end{aligned}
$$


and

$$
\begin{aligned}
\int_{0}^{\infty} A(\xi) \sin \xi y d \xi & =D_{1} e^{\lambda_{1} y}+D_{2} e^{\bar{\lambda}_{1} y}+D_{3} e^{\lambda y}+R \frac{e^{-\lambda y}}{Q(\lambda)} \\
& \equiv h(y) \quad \text { for } y \in(b, \infty)
\end{aligned}
$$

where $C_{1}, C_{2}, C_{3}, D_{1}, D_{2}$ and $D_{3}$ are arbitrary constants.

In order to accommodate zero and the point at infinity along the $y$-axis, $D_{3}$ in (3.27) is taken to be zero and the function $f(y)$ must satisfy $f(0)=f^{\prime \prime}(0)=0$, that is,

$$
C_{1}+C_{2}+C_{3}-\frac{i \lambda}{Q(\lambda)} R=-\frac{i \lambda}{Q(\lambda)}
$$

and

$$
\lambda^{2} C_{1}+\lambda_{1}^{2} C_{2}+\bar{\lambda}_{1}^{2} C_{3}-\frac{i \lambda^{3}}{Q(\lambda)} R=-\frac{i \lambda^{3}}{Q(\lambda)}
$$

Defining

$$
\int_{0}^{\infty} \xi A(\xi) \sin \xi y d \xi=g(y) \text { for } y \in(b, \infty)
$$

and making use of relation (3.26), we obtain

$$
A(\xi)=\frac{2}{\pi \xi} \int_{0}^{\infty} P(y) \sin \xi y d y \quad \text { where } P(y)= \begin{cases}f(y), & \text { for } y \in(0, b), \\ g(y), & \text { for } y \in(b, \infty) .\end{cases}
$$

By putting $A(\xi)$ from (3.30) into relation (3.27), we derive that the unknown function $g(y)$ satisfies the weakly singular integral equation

$$
\frac{1}{\pi} \int_{b}^{\infty} g(u) \log \left|\frac{u+x}{u-x}\right| d u=h_{1}(x) \quad \text { for } x \in(b, \infty)
$$

where

$$
h_{1}(x)=h(x)-\frac{1}{\pi} \int_{0}^{b} f(t) \log \left|\frac{x+t}{x-t}\right| d t .
$$

Differentiating relation (3.26) and $g(y)$ twice and, again by application of the Fourier sine transform, we obtain that

$$
\xi^{2} A(\xi)=-\frac{2}{\pi \xi} \int_{0}^{\infty} \frac{d^{2} P}{d y^{2}} \sin \xi y d y,
$$

where the function $g_{1}=d^{2} g / d y^{2}$ satisfies the weakly singular integral equation

$$
\frac{1}{\pi} \int_{b}^{\infty} g_{1}(u) \log \left|\frac{u+x}{u-x}\right| d u=h_{2}(x) \quad \text { for } x \in(b, \infty),
$$

with

$$
h_{2}(x)=\frac{d^{2} h}{d x^{2}}-\frac{1}{\pi} \int_{b}^{\infty} \frac{d^{2} f}{d t^{2}} \log \left|\frac{x+t}{x-t}\right| d t .
$$


Following a similar argument to the earlier case, one can prove that $g(y)$ and $d^{2} g / d y^{2}$ are bounded at the end point $y=b$ and the behaviour is

$$
\frac{d^{2} g}{d y^{2}} \sim O\left(|y-t|^{1 / 2}\right) \quad \text { as } y \rightarrow b^{+} .
$$

Hence the bounded solutions for (3.31) and (3.33) are given by

$$
g(u)=\frac{2}{\pi} \frac{\sqrt{u^{2}-b^{2}}}{u^{2}} \int_{b}^{\infty} \frac{t h_{1}^{\prime}(t)}{\sqrt{t^{2}-b^{2}}\left(t^{2}-u^{2}\right)} d t \quad u \in(b, \infty),
$$

provided that

$$
\int_{b}^{\infty} \frac{t h_{1}^{\prime}(t)}{\sqrt{t^{2}-b^{2}}} d t=0
$$

and

$$
g_{1}(u)=\frac{d^{2} g}{d u^{2}}=\frac{2}{\pi} \frac{\sqrt{u^{2}-b^{2}}}{u^{2}} \int_{b}^{\infty} \frac{t h_{2}^{\prime}(t)}{\sqrt{t^{2}-b^{2}}\left(t^{2}-u^{2}\right)} d t \quad u \in(b, \infty),
$$

provided that

$$
\int_{b}^{\infty} \frac{t h_{2}^{\prime}(t)}{\sqrt{t^{2}-b^{2}}} d t=0
$$

Utilizing the integrals in the Appendix, we express conditions (3.34) and (3.36) in terms of unknown constants by

$$
\begin{aligned}
& J_{4}(\lambda) C_{1}+J_{4}\left(\lambda_{1}\right) C_{2}+J_{4}\left(\bar{\lambda}_{1}\right) C_{3}-b \lambda_{1} K_{1}\left(-b \lambda_{1}\right) D_{1}-b \bar{\lambda}_{1} K_{1}\left(-b \bar{\lambda}_{1}\right) D_{2} \\
& \quad-\left[\frac{i \lambda}{Q(\lambda)} J_{4}(-\lambda)-\frac{b \lambda}{Q(\lambda)} K_{1}(b \lambda)\right] R=-\frac{i \lambda}{Q(\lambda)} J_{4}(-\lambda)
\end{aligned}
$$

and

$$
\begin{gathered}
\lambda^{2} J_{4}(\lambda) C_{1}+\lambda_{1}^{2} J_{4}\left(\lambda_{1}\right) C_{2}+\bar{\lambda}_{1}^{2} J_{4}\left(\bar{\lambda}_{1}\right) C_{3}-b \lambda_{1}^{3} K_{1}\left(-b \lambda_{1}\right) D_{1}-b \bar{\lambda}_{1}^{3} K_{1}\left(-b \bar{\lambda}_{1}\right) D_{2} \\
-\left[\frac{i \lambda^{3}}{Q(\lambda)} J_{4}(-\lambda)-\frac{b \lambda^{3}}{Q(\lambda)} K_{1}(b \lambda)\right] R=-\frac{i \lambda^{3}}{Q(\lambda)} J_{4}(-\lambda),
\end{gathered}
$$

where $K_{1}(x)$ is the modified Bessel function and $J_{i}(x), i=1,2,3,4$, are given in the Appendix.

Also, by matching relations (3.30) and (3.32), conditions are obtained for the functions $g$ and $h$ :

$$
f(b)=0 \text { that is, } C_{1} e^{\lambda b}+C_{2} e^{\lambda_{1} b}+C_{3} e^{\bar{\lambda}_{1} b}-R \frac{i \lambda}{Q(\lambda)} e^{-\lambda b}=-\frac{i \lambda}{Q(\lambda)} e^{-\lambda b}
$$

and

$$
g^{\prime}(b)=f^{\prime}(b)
$$


It may be remarked here that it is not straightforward, as in the case of submerged structure, to modify relation (3.40) for its suitable form by integrating the solution of the integral equation (3.35). Instead, relation (3.27) may be differentiated twice and integrated from $b$ to $\infty$ to get

$$
\int_{0}^{\infty} \xi^{2} A(\xi) J_{0}(\xi b) d \xi=-\int_{b}^{\infty} \frac{h^{\prime \prime}(y)}{\sqrt{y^{2}-b^{2}}} d y .
$$

Utilizing the expression for $\xi^{2} A(\xi)$ from (3.32) and relation (3.40), we finally arrive at

$$
\int_{b}^{\infty} \frac{h^{\prime \prime}(y)}{\sqrt{y^{2}-b^{2}}} d y+f^{\prime}(0)=0,
$$

which is expressed in terms of the unknown constants as

$$
\begin{aligned}
& \lambda C_{1}+\lambda_{1} C_{2}+\bar{\lambda}_{1} C_{3}+\lambda_{1}^{2} K_{0}\left(-b \lambda_{1}\right) D_{1}+\bar{\lambda}_{1}^{2} K_{0}\left(-b \bar{\lambda}_{1}\right) D_{2} \\
& +\left[\frac{i \lambda^{2}}{Q(\lambda)}\left(1-i K_{0}(b \lambda)\right)\right] R=\frac{i \lambda^{2}}{Q(\lambda)} .
\end{aligned}
$$

This completes the description involved in the determination of the analytical solution of the general mixed boundary value problem posed in Section 2, corresponding to the case of the surface piercing vertical barrier. Hence, all of the unknown constants can be obtained from relations (3.28), (3.29), (3.37)-(3.39) and (3.41).

3.3. Numerical results The unknown constants $C_{1}, C_{2}, C_{3}, D_{1}, D_{2}$ and $R$ can be analytically determined from relations (3.6), (3.7), (3.18), (3.20), (3.21) and (3.23) for the submerged case, and from relations (3.28), (3.29), (3.37)-(3.39) and (3.41) for the surface piercing case. The absolute values of the reflection coefficient $|R|$ are plotted in Mathematica for different values of a nondimensional membrane tension parameter $\beta=M K^{2}$ against a nondimensional wave parameter $\alpha=K b$ for each of the barrier configurations.

The membrane coupled gravity wave propagation exists purely for $M \neq 0$ and does not exist when the membrane tension is ignored, that is, $M=0$. However, one might expect that the numerically calculated values for very small $M$ would match with the exact values in the limiting case. In the case of a submerged barrier, exact values of the reflection coefficient for free surface gravity waves are given by

$$
R=\frac{K_{0}(K b)}{K_{0}(K b)+i I_{0}(K b)} .
$$

The graphical profiles of the reflection coefficient $|R|$, for a submerged barrier, are plotted in Figures 2-5 for various $\beta$ values. In Figure 2, the thin line denotes the reflection coefficient curve that is computed for membrane coupled waves by choosing a very small $\beta$ parameter (say, $10^{-5}$ ), while the dashed line denotes the exact reflection coefficient curve applicable for free surface gravity waves. Hence, the numerical results for a very small value membrane parameter agree with the analytical results pertaining to free surface gravity waves. 


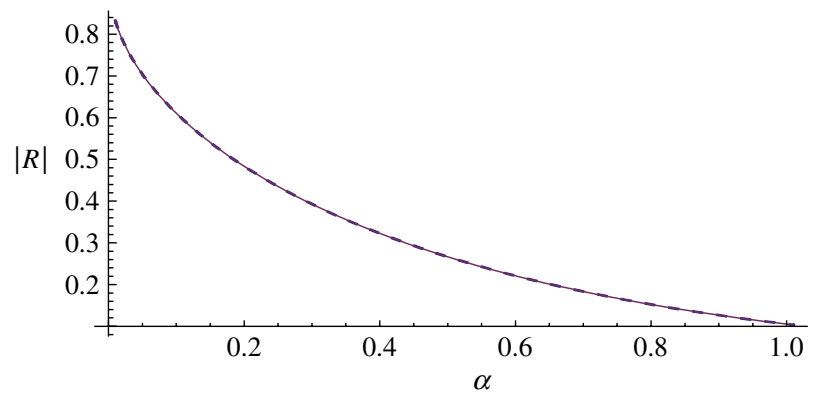

FIGURE 2. Reflection coefficient $|R|$ for submerged vertical barrier plotted against the nondimensional wavenumber $\alpha$. The dashed line shows the results for $\beta=0$ and the solid line shows those for $\beta=10^{-5}$.

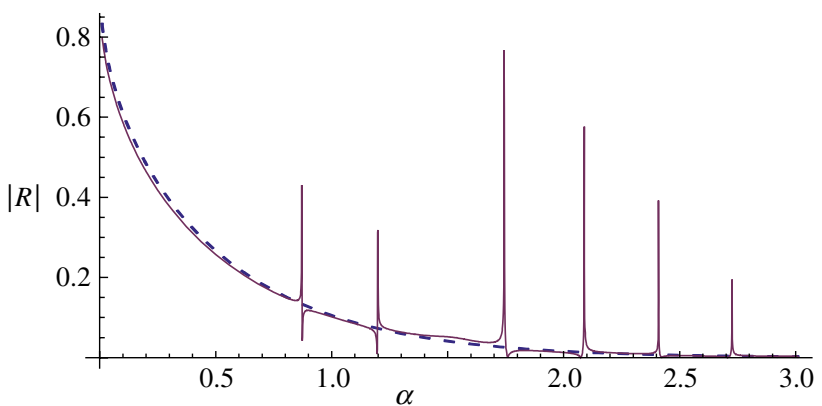

(a)

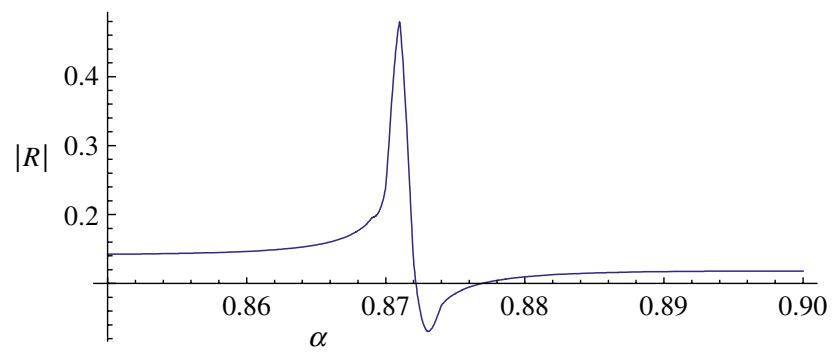

(b)

FIgURE 3. (a) Reflection coefficient $|R|$ for a submerged vertical barrier plotted against the nondimensional wavenumber $\alpha$. The dashed line shows results for $\beta=0$ and the solid line shows those for $\beta=0.01$. (b) Window around a spike in $|R|$ for $\beta=0.01$.

With an increase in the value of the tension parameter, say, $\beta=0.01$, at certain frequencies, there is a sharp increase in the reflection coefficient. This is an interplay between membrane tension and incident wave frequency. These isolated frequencies are called "resonant frequencies" in the literature. Such a phenomenon was also observed for free surface gravity wave scattering by a complete vertical membrane barrier (see [8]). Also numerical computations, for different values of $\beta$, suggest that 


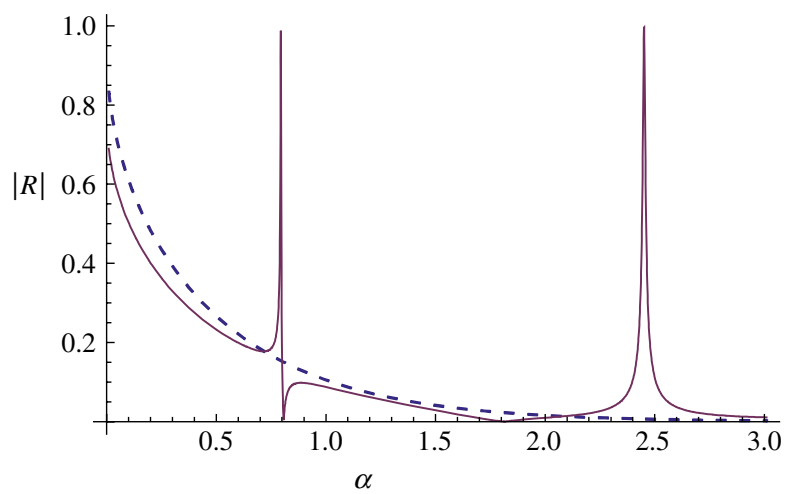

FIGURE 4. Reflection coefficient $|R|$ for a submerged vertical barrier plotted against the nondimensional wavenumber $\alpha$. The dashed line shows results for $\beta=0$ and the solid line shows those for $\beta=0.1$.

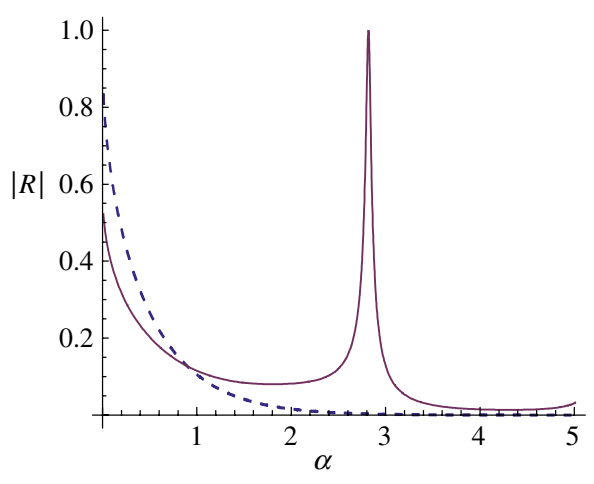

(a)

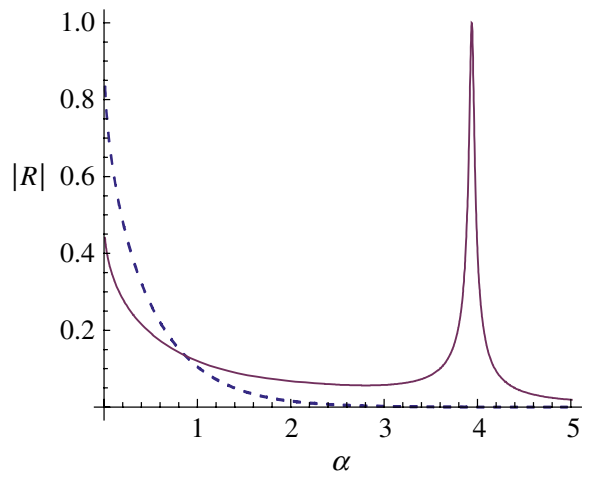

(b)

FIGURE 5. Reflection coefficient $|R|$ for a submerged vertical barrier plotted against the nondimensional wavenumber $\alpha$. The dashed line shows results for $\beta=0$ and the solid line shows those for (a) $\beta=0.5$ and (b) $\beta=1.0$.

these frequencies start widening and the gap between two successive frequencies increases with an increase in the membrane tension parameter. Naturally, one might attribute these sharp increases in the reflection curve to the natural frequencies of the membrane under tension.

The graphs for the reflection coefficient $|R|$, for different $\beta$ values, are shown in Figures $3-5$ in which the thin line represents the computed values while dashes denote the exact value at $\beta=0$. A typical spike in the reflection curve for $\beta=0.01$ is shown in Figure 3(b). For $\beta=0.1,0.5,1.0$, values of the reflection coefficient are smaller than those for free surface waves in the specific lower frequency range. As $\alpha$ becomes bigger, these values catch up with the free surface reflection curve and rise sharply at resonant frequencies. Overall, they are a little higher than the free surface reflection values at larger $\alpha$ as shown in Figures 4 and 5. 


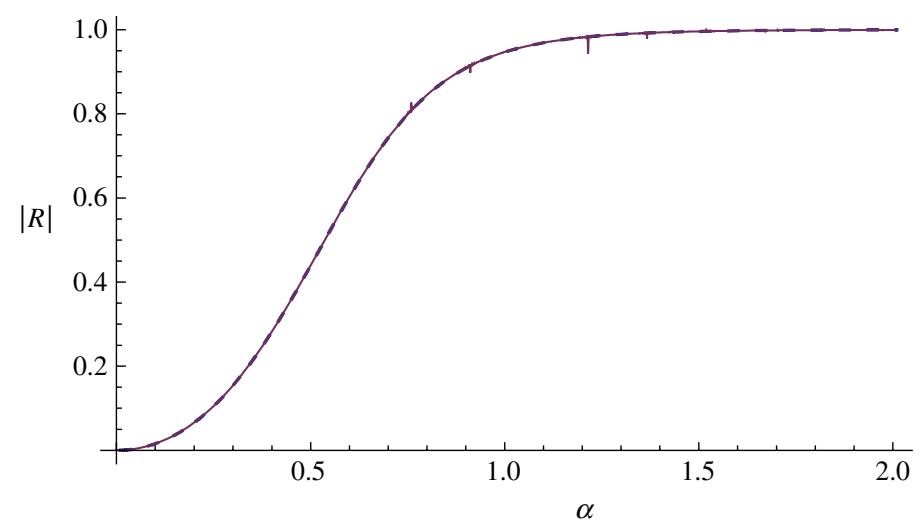

(a)

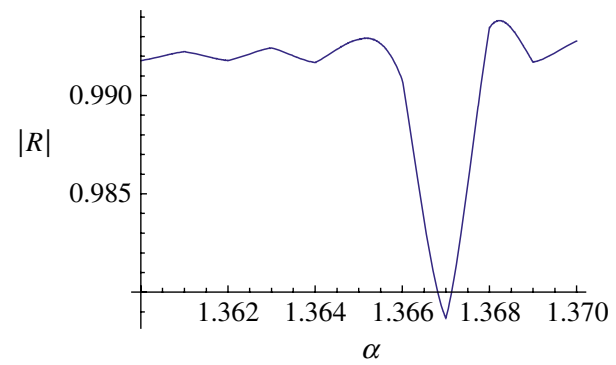

(b)

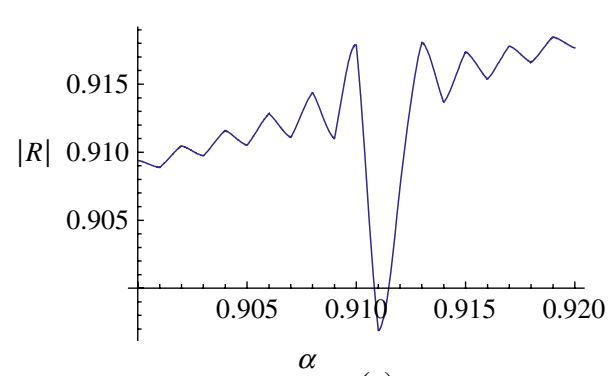

(c)

FIGURE 6. Reflection coefficient $|R|$ for a surface piercing vertical barrier plotted against the nondimensional wavenumber $\alpha$. (a) The dashed line shows results for $\beta=0$ and the solid line shows those for $\beta=10^{-7}$; (b), (c) show windows around the spikes of $|R|$ for $\beta=10^{-7}$.

Numerical results clearly suggest that the submerged vertical barrier performs well as a wave barrier. Its performance depends on the amount of reflection it offers. For small membrane tension, there appear to be many resonant frequencies at which sharp reflection occurs. However, for bigger values of $\beta$, the length of the gap $b$ can be suitably chosen to allow the resonant frequencies to fall within a fixed range of wave frequencies, since $\alpha=\omega^{2} b / g$. As resonant frequencies move towards the right at higher membrane tension, for better performance of the submerged wave barrier, one may require a bigger (or a smaller) value of $b$ for short (or long) incident waves. Finally, energy conservation $|R|^{2}+|T|^{2}=1$, with $T=1-R$, is verified numerically for a submerged barrier.

In Figures 6 and 7, the reflection coefficients $|R|$ for a surface piercing barrier are plotted against the parameter $\alpha$ for different values of $\beta$. Also, for the prescribed edge conditions, there is no energy dissipation as shown in [6], that is, $|R|^{2}+|T|^{2}=1$. This would imply that there is a conservation of energy in this case too, and this has been verified numerically. However, the reflection curves appear to have negligible spikes 


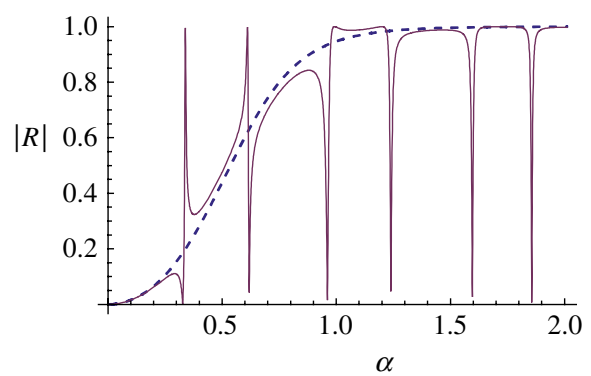

(a)

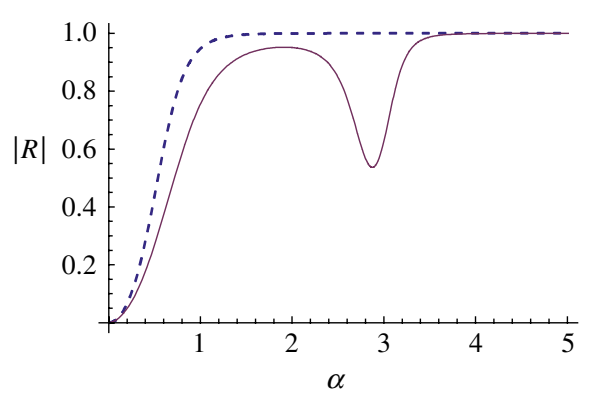

(c)

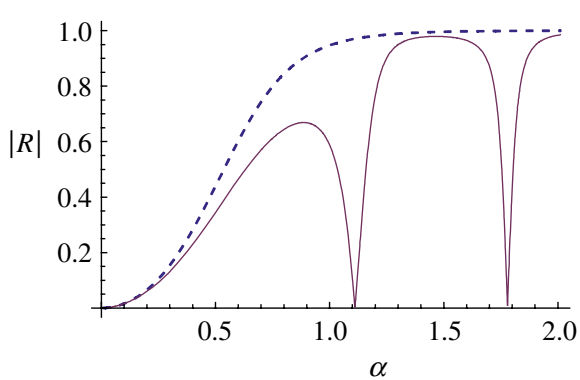

(b)

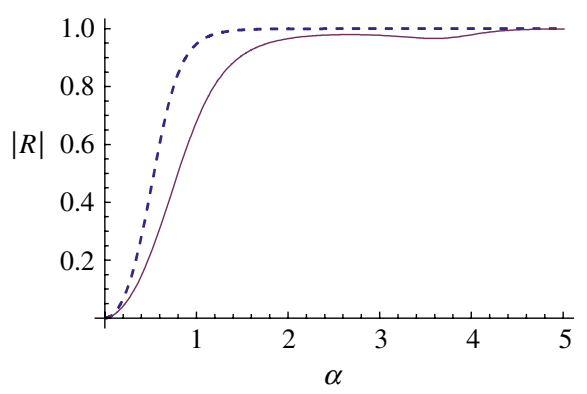

(d)

FIGURE 7. Reflection coefficient $|R|$ for a surface piercing vertical barrier plotted against the nondimensional wavenumber $\alpha$. (a) The dashed line shows results for $\beta=0$ and the solid line shows those for (a) $\beta=0.01$, (b) $\beta=0.1$, (c) $\beta=0.5$ and (d) $\beta=1.0$.

for small values of $\beta$ closer to zero. For example, when $\beta=10^{-7}$, the membrane surface reflection curve is shown in Figure 6(a) along with the free surface reflection curve which is computed from the analytical result

$$
R=\frac{\pi I_{1}(K b)}{\pi I_{1}(K b)+i K_{1}(K b)} .
$$

Also, these spike-like variations are shown in the Figures 6(b) and 6(c). It is worth noting that these negligible spiky variations are not numerical discrepancies but are the effect of very small membrane tension on waves interacting with the surface piercing barrier. However, these spikes vanish when $\beta$ is further reduced to $10^{-9}$.

The reflection coefficients are also computed for $\beta=0.01,0.1,0.5$ and 1.0; these are shown in Figure 7. Contrary to the submerged barrier case, it is observed that there are sharp dips, at certain resonant frequencies, in the reflection coefficient for each specified $\beta$. For small $\beta$ values, say 0.01 , there are also a few sharp rises in the reflection curve at the lower frequencies. As the value of the membrane tension parameter increases, the positions of these sharp dips or resonant frequencies move to the right and the gap between two successive dips increases. In the process, sharp rises 
start disappearing and one can see only dips or zero reflection values at all the resonant frequencies.

Physically, for these wave frequencies, either reflection or transmission occurs partially or completely. In other words, the progressive wave gets trapped on either side of the partial barrier. In designing wave barriers, these frequencies must be considered and avoided if necessary. When the membrane tension is high, these dips are greatly reduced and the reflection is not as high as for free surface gravity waves.

Finally, it may be remarked that this kind of trapping phenomenon has also been studied numerically (see $[4,17]$ ) for gravity waves at the end of a semi-infinitely long channel of finite depth, with either rigid or porous type partial vertical barriers.

\section{Conclusions}

In the present study, scattering of membrane coupled gravity water waves by partial vertical barriers is considered. Using certain mode-coupling relations, the corresponding mixed boundary value problem has been reduced to the problem of solving a set of dual integral equations. These equations are solved by converting them into a pair of singular integral equations, with logarithmic kernel. The end behaviour of solutions of these weakly singular integral equations forces a mathematical constraint which helps in determining the unknowns associated with the dual integral equations analytically. The graphical profiles of the reflection coefficients have been plotted against a nondimensional wave parameter for different values of the nondimensional membrane tension. In the limiting case $M \rightarrow 0$ and $M \neq 0$, numerical results for the reflection and transmission coefficients match exactly with exact values for the free surface gravity waves. Also, when $M \neq 0$, there exist resonant frequencies at which either sharp rises or dips (or both) in reflection coefficient were observed. This does not happen for the free surface gravity scattering by these structures. The present method of solution can be extended to mixed boundary value problems corresponding to the scattering of more general flexural gravity waves by partial vertical barriers as well as by a vertical barrier with a finite number of gaps in it. Further, the solution procedure applied here to solve a set of dual integral equations with trigonometric kernel is quite useful for dealing with similar boundary value problems in other branches of science and engineering.

\section{Acknowledgements}

The author thanks the Associate Editor Graeme Hocking and the referees for their valuable comments which improved the presentation of this paper.

\section{Appendix. Standard integrals}

In this appendix, we list certain integrals evaluated by standard contour integration technique and a few among the other integrals utilized in the analysis with notation $J_{i}(x), i=1,2,3,4$. The reader may refer to Equations $3.387(5,6), 3.389(3,4)$, 
3.753(3) and 6.693(7) in Gradshteyn and Ryzhik [7].

$$
\begin{array}{ccc}
\int_{0}^{b} \frac{d t}{\sqrt{b^{2}-t^{2}\left(x^{2}-t^{2}\right)}}=\frac{\pi}{2 x \sqrt{x^{2}-b^{2}}} & \text { for } x>b, \\
\int_{b}^{\infty} \frac{t}{\sqrt{t^{2}-b^{2}\left(t^{2}-x^{2}\right)} d t}=\frac{\pi}{2 \sqrt{b^{2}-x^{2}}} \quad \text { for } x<b, \\
\int_{0}^{b} \frac{t^{2} \sqrt{b^{2}-t^{2}}}{\left(t^{2}-x^{2}\right)} d t=-\frac{\pi}{4}\left[2 x^{2}-1\right] & \text { for } 0<x<b, \\
\int_{0}^{b} \frac{t^{2}}{\sqrt{b^{2}-t^{2}}\left(t^{2}-x^{2}\right)} d t=-\frac{2}{\pi}\left[\frac{x}{\sqrt{x^{2}-b^{2}}}-1\right] & \text { for } x>b, \\
J_{1}(x)=\frac{\pi}{2}\left[I_{0}(b x)+L_{0}(b x)\right], & b^{2} \pi \\
J_{2}(x)=\frac{b \pi}{2}\left[I_{0}(b x)+L_{0}(b x)\right]-\frac{b \pi}{2 x}\left[I_{1}(b x)+L_{1}(b x)\right], & \\
J_{3}(x)=-\frac{b}{x} K_{1}(-b x)+b^{2} K_{0}(-b x), & J_{4}(x)=b\left[1+\frac{\pi}{2}\left(I_{1}(x b)+L_{1}(x b)\right)\right]
\end{array}
$$

where $K_{0}, K_{1}, I_{0}$ and $I_{1}$ are modified Bessel functions and $L_{0}$ and $L_{1}$ are Struve functions.

\section{References}

[1] A. Chakrabarti and S. R. Manam, "Solution of the logarithmic singular integral equation", Appl. Math. Lett. 16 (2003) 369-373.

[2] I. H. Cho and M. H. Kim, "Interaction of a horizontal flexible membrane with oblique incident waves", J. Fluid Mech. 367 (1998) 139-161.

[3] I. H. Cho and M. H. Kim, "Wave deformation by a submerged circular disk", Appl. Ocean Res. 21 (1999) 263-280.

[4] A. T. Chwang and Z. N. Dong, "Wave trapping due to a porous plate", Proceedings of the 15th ONR Symposium on Naval Hydrodynamics, (National Academy Press, Washington, DC, 1984) 407-414.

[5] R. Estrada and R. P. Kanwal, "Integral equations with logarithmic kernels", IMA J. Appl. Math. 43 (1989) 133-155.

[6] D. V. Evans, "The influence of surface tension on the reflection of water waves by a plane vertical barrier", Proc. Cambridge Philos. Soc. 64 (1968) 795-810.

[7] I. S. Gradshteyn and I. M. Ryzhik, Table of integrals, series and products (Academic Press, London, 1980).

[8] M. H. Kim and S. T. Kee, "Flexible membrane wave barrier. I: Analytic and numerical solutions", ASCE J. Waterway, Port, Coastal Ocean Engng 122 (1996) 46-53.

[9] S. R. Manam, J. Bhattacharjee and T. Sahoo, "Expansion formulae in wave structure interaction problems", Proc. R. Soc. Lond. A 462 (2006) 263-287.

[10] P. F. Rhodes-Robinson, "The effect of surface tension on the progressive waves due to incomplete vertical wave-makers in water of infinite depth", Proc. R. Soc. Lond. A 435 (1991) 293-319. 
[11] T. Sahoo, T. L. Yip and A. T. Chwang, "Wave interaction with a semi-infinite horizontal membrane", Proceedings of XXIX IAHR Congress, Theme E-Hydraulics for Maritime Engineering, Beijing, China, 2001, (Tsinghua University Press, Beijing, 2001) 109-117.

[12] T. Sawaragi, Coastal engineering-waves, beaches, wave-structure interactions (Elsevier, Tokyo, 1995).

[13] J. J. Stoker, "Surface waves in water of variable depth", Quart. Appl. Math. 5 (1947) 1-54.

[14] F. Ursell, "The effect of a fixed vertical barrier on surface waves in deep water", Proc. Cambridge Philos. Soc. 43 (1947) 374-382.

[15] W. E. Williams, "A note on scattering of water waves by a vertical barriers", Proc. Cambridge Philos. Soc. 62 (1966) 507-509.

[16] T. L. Yip, T. Sahoo and A. T. Chwang, "Wave scattering by multiple floating membranes", Proceedings of the 11th International Offshore and Polar Engineering Conference, Stavangar, Norway, 2001, (International Society of Offshore and Polar Engineers, Cupertino, CA, 2001) 3, 379-384.

[17] T. L. Yip, T. Sahoo and A. T. Chwang, "Trapping of surface waves by porous and flexible structures", Wave Motion 35 (2002) 41-54. 